\title{
Cell Death after Spinal Cord Injury Is Exacerbated by Rapid TNF $\alpha$-Induced Trafficking of GluR2-Lacking AMPARs to the Plasma Membrane
}

\author{
Adam R. Ferguson, ${ }^{1}$ Randolph N. Christensen, ${ }^{4}$ John C. Gensel, ${ }^{3}$ Brandon, A. Miller, ${ }^{1,2}$ Fang Sun, ${ }^{6}$ Eric C. Beattie, ${ }^{5}$ \\ Jacqueline C. Bresnahan, ${ }^{1}$ and Michael S. Beattie ${ }^{1}$ \\ ${ }^{1}$ Brain and Spinal Injury Center, Department of Neurological Surgery, University of California, San Francisco, San Francisco, California 94110, ${ }^{2}$ Department \\ of Neuroscience and ${ }^{3}$ Department of Molecular Virology, Immunology, and Medical Genetics, Center for Brain and Spinal Repair, The Ohio State \\ University, Columbus, Ohio 43210, ${ }^{4}$ Department of Biology, Coe College, Cedar Rapids, Iowa 52402, ${ }^{5}$ Department of Neurosciences, California Pacific \\ Medical Center Research Institute, San Francisco, California 94107, and `Neurology, Harvard University, Children’s Hospital, Boston, Massachusetts 02115
}

Glutamate, the major excitatory neurotransmitter in the CNS, is implicated in both normal neurotransmission and excitotoxicity. Numerous in vitro findings indicate that the ionotropic glutamate receptor, AMPAR, can rapidly traffic from intracellular stores to the plasma membrane, altering neuronal excitability. These receptor trafficking events are thought to be involved in CNS plasticity as well as learning and memory. AMPAR trafficking has recently been shown to be regulated by glial release of the proinflammatory cytokine tumor necrosis factor $\alpha(\mathrm{TNF} \alpha)$ in vitro. This has potential relevance to several CNS disorders, because many pathological states have a neuroinflammatory component involving TNF $\alpha$. However, TNF $\alpha$-induced trafficking of AMPARs has only been explored in primary or slice cultures and has not been demonstrated in preclinical models of CNS damage. Here, we use confocal and image analysis techniques to demonstrate that spinal cord injury (SCI) induces trafficking of AMPARs to the neuronal membrane. We then show that this effect is mimicked by nanoinjections of TNF $\alpha$, which produces specific trafficking of GluR2-lacking receptors which enhance excitotoxicity. To determine if TNF $\alpha$-induced trafficking affects neuronal cell death, we sequestered TNF $\alpha$ after SCI using a soluble TNF $\alpha$ receptor, and significantly reduced both AMPAR trafficking and neuronal excitotoxicity in the injury penumbra. The data provide the first evidence linking rapid TNF $\alpha$-induced AMPAR trafficking to early excitotoxic secondary injury after CNS trauma in vivo, and demonstrate a novel way in which pathological states hijack mechanisms involved in normal synaptic plasticity to produce cell death.

Key words: inflammation; excitotoxicity; trauma; plasticity; neuroinflammation; neural-immune interaction; glia-neuron interactions

\section{Introduction}

Glutamate receptors can be rapidly inserted or deleted from neuronal membranes, thereby affecting synaptic function (Malinow and Malenka, 2002). Trafficking of AMPA receptors (AMPARs) has been shown to be affected by synaptic activation, and in cultured hippocampal neurons appears also to be regulated by nonsynaptic events, including the release of insulin (Skeberdis et al., 2001), cholesterol (Hering et al., 2003), and glial tumor necrosis factor $\alpha$ (TNF $\alpha$ ) (Beattie et al., 2002). This has provided support to the concept that glial cells and circulating cytokines can affect

Received Aug. 4, 2008; accepted Sept. 23, 2008.

This work was supported by National Institutes of Health Grant R01 NS38079 (J.C.B., M.S.B.), New York State CoRE (Center of Research Excellence) (M.S.B., J.C.B.), and Christopher Reeve Foundation Grants BB-0204-2 (M.S.B.), R01 MH067931 (E.C.B.), F32 NS053059 (A.R.F.), F32 NS045468 (R.N.C.), and F30 NS053185 (B.A.M.). We thank C. Amy Tovar and Rochelle Deibert for technical and surgical expertise, Yvette $S$. Nout and Karen-Amanda Irvine for surgical expertise, John Komon for help with figures, Ohio State University Campus Microscopy and Imaging Facility, and Dmitri Leonoudakis for helping develop the image analysis techniques. We thank Karen-Amanda Irvine and Sergio Veiga for helpful comments on an earlier version of this manuscript.

Correspondence should be addressed to Dr. Michael S. Beattie, Department of Neurological Surgery, Brain and Spinal Injury Center, University of California, San Francisco, 1001 Potrero Avenue, Building 1, Room 101, San Francisco, CA 94110. E-mail: Michael.Beattie@ucsf.edu.

DOI:10.1523/JNEUROSCI.3708-08.2008

Copyright $\odot 2008$ Society for Neuroscience ～0270-6474/08/2811391-10\$15.00/0 synaptic regulation (Stellwagen and Malenka, 2006). TNF $\alpha$ and other cytokines are released in response to stress and after injury, suggesting that these pathological situations may also affect synaptic transmission. Furthermore, because AMPARs are implicated in excitotoxicity, injury-induced TNF $\alpha$ release could exacerbate cell death by the same mechanisms that control glutamatergic synaptic strength, i.e., receptor trafficking. This notion is supported by findings that coinjection of TNF $\alpha$ with low doses of the glutamate agonist kainic acid produce marked cell death in spinal neurons in vivo (Hermann et al., 2001). In addition, in hippocampal cultures TNF $\alpha$ increases GluR2lacking AMPAR surface expression (Stellwagen et al., 2005), thereby enhancing $\mathrm{Ca}^{2+}$ influx (Leonoudakis et al., 2008) and providing a potential mechanism for the observed increase in excitotoxicity in vivo. The present study was aimed at determining whether rapid AMPAR trafficking occurs as part of the pathological sequelae in spinal cord injury (SCI), if it is mediated by TNF $\alpha$, and whether blocking TNF $\alpha$ could reduce cell death.

AMPAR trafficking has been studied in vitro using electrophysiology (Adesnik et al., 2005), live cell imaging (SekineAizawa and Huganir, 2004) and cell surface biotinylation (Ehlers, 2000); these methods allow for separation of populations of re- 
ceptors exposed to the extracellular space from those in intracellular stores. Measuring trafficking in vivo is more challenging because many of the above approaches cannot be used. However, if examinations of this and similar hypotheses are to be moved from the in vitro situation to the more translational setting of the intact adult CNS, such measurements will be necessary. In the present study, we tested for TNF $\alpha$-induced AMPAR trafficking in preclinical models of SCI by combining whole tissue membrane fractionation and Western blotting with optical methods using high-resolution confocal microscopy, blind iterative deconvolution, and automated image analysis techniques on fixed tissue. Laser scanning confocal data revealed that SCI significantly increases synaptic AMPARs in spinal neurons in vivo, suggesting increased excitotoxic potential. Biochemical fractionation and confocal techniques demonstrated that nanoinjections of TNF $\alpha$ also induce a rapid, dosedependent increase in GluR2-lacking AMPARs in the neuronal plasma membrane at both extrasynaptic and synaptic sites. Finally, we show that sequestration of TNF $\alpha$ with a soluble form of human recombinant TNF $\alpha$ receptor 1 after spinal cord injury reduces both AMPAR trafficking to the membrane and acute neuronal excitotoxicity. These findings suggest that injuryinduced increases in TNF $\alpha$ alter AMPAR trafficking and contribute to excitotoxic cell death via a novel process that involves the hijacking of mechanisms involved in the modulation of normal synaptic transmission.

\section{Materials and Methods}

Animals. Subjects were 77- to 87-d-old female Long-Evans rats that were housed as pairs in Association for Assessment and Accreditation of Laboratory Animal Care-approved facilities at Ohio State University and the University of California, San Francisco. All experiments were performed in accordance with National Institutes of Health guidelines and were approved by Institutional Animal Care and Use Committees at The Ohio State University and the University of California, San Francisco.

Cervical spinal cord injury. Subjects were anesthetized with pentobarbital and a dorsal midline incision was made. Connective tissue was dissected and a C5 laminectomy was performed. Subjects were given unilateral cervical contusion injuries using a New York University/ Multicenter Animal Spinal Cord Injury Study device with a $2.0 \mathrm{~mm}$ head dropped from an impact height of $6.25 \mathrm{~mm}$. These procedures have been shown to produce a focal lesion with minimal contralateral spread, allowing use of the contralateral side as an internal control (Gensel et al., 2006). Given the regional effect of injury, microscope field was used as the unit of analysis, yielding sample sizes of $n=17$ for spinal cord injury and $n=21$ for control. To provide an accurate estimate of total synaptic AMPAR, each field was sampled in three-dimensions (3D) with a laser scanning microscope (yielding 608 total images) and quantification was performed on the mean three-dimensional colocalization for each field. The experiment was replicated at $90 \mathrm{~min}$ and $3 \mathrm{~h}$ to provide a time course analysis ( $n=4$ subjects with SCI tested as a within-subject variable) (Fig. 1).

$T N F \alpha$ nanoinjection. Subjects were given stereotactic nanoinjections ( $35 \mathrm{nl}$ ) into the T9-10 ventral horn as previously described (Hermann et al., 2001) using compressed air micropressure applied to pulled glass pipettes (tip diameter, $30 \mu \mathrm{m}$; $30^{\circ}$ bevel; Radnoti). Albumin $(1 \mu \mathrm{M})$ was used as a control because it has molecular weight similar to rat recombinant TNF $\alpha$ (R\&D systems). For biochemical experiments, subjects ( $n=$ 5) were given nanoinjections of either $\operatorname{TNF} \alpha(1 \mu \mathrm{M})$ or albumin into four evenly spaced sites within a $750 \mu \mathrm{m}$ length of spinal cord (Fig. $2 \mathrm{~A}$; supplemental Fig. 1, available at www.jneurosci.org as supplemental material). For imaging experiments, subjects $(n=4)$ received a single TNF $\alpha$ dose $(0.01,0.1$, or $1 \mu \mathrm{M})$, and each subject received a contralateral control injection of albumin (supplemental Fig. 2A, available at www. jneurosci.org as supplemental material). The selected TNF $\alpha$ doses have been shown to be insufficient to induce cell death alone but enhance glutamate-mediated excitotoxicity in the spinal cord (Hermann et al., 2001). To localize injections (supplemental Fig. $2 B$, available at www. jneurosci.org as supplemental material), FluoroRuby (Invitrogen) was included in the solutions.

Nanoinjection of soluble TNF $\alpha$ receptor 1 after cervical SCI. Subjects were given unilateral cervical spinal cord injuries as described above and then placed in a spinal stereotaxic for intraparenchymal treatment with human recombinant soluble TNF $\alpha$ receptor (R\&D systems). The dura was opened under a dissecting scope and then two nanoinjection pipettes (50 $\mu \mathrm{m}$ tip diameter, $30^{\circ}$ bevel, Radnoti) were positioned under stereotaxic control to target the rostral and caudal edges of the lesion. Pipettes were mounted on a stereotaxic arm at a fixed space of $7.2 \mathrm{~mm}$ apart, with the visible contusion hematoma placed equidistant between the tips. Drug or vehicle was delivered into the spinal parenchyma at a depth of 1.4 $\mathrm{mm}$ at the midline, at a dose of $10 \mu \mathrm{g} / \mathrm{ml}$ dissolved in PBS containing $0.1 \%$ BSA. Solutions were delivered in $30 \mathrm{nl}$ bursts over $5 \mathrm{~min}$ per pipette for $60 \mathrm{~min}$ (subjects were killed $90 \mathrm{~min}$ after injury), resulting in a total dose of $7.2 \mathrm{ng}$ soluble TNF $\alpha$ receptor 1 (sTNF $\alpha \mathrm{R} 1)$ in $720 \mathrm{nl}$ of solution per subject. To achieve a balanced design, nanoinjection of sTNF $\alpha$ R1 was compared against two control groups: vehicle nanoinjection, and a noninjected contusion lesion $(n=4)$.

Subcellular fractionation. Sixty minutes after TNF $\alpha$ or albumin nanoinjections, $7.5 \mathrm{~mm}$ of spinal cord was extracted under deep anesthesia, snap-frozen on dry ice, and placed in a $-80^{\circ} \mathrm{C}$ freezer for later processing. The fractionation procedures were adapted from previous work with mouse spinal cord (Galan et al., 2004). Frozen spinal cord was 
A

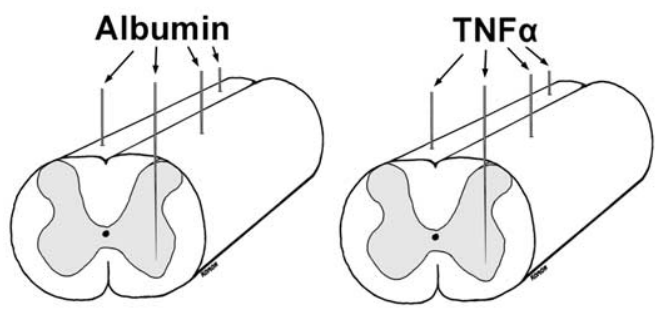

B

\section{Plasma Membrane Enrichment by Centrifugation}
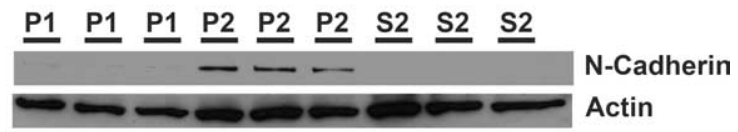

C

AMPAR Levels in Plasma Membrane Fraction

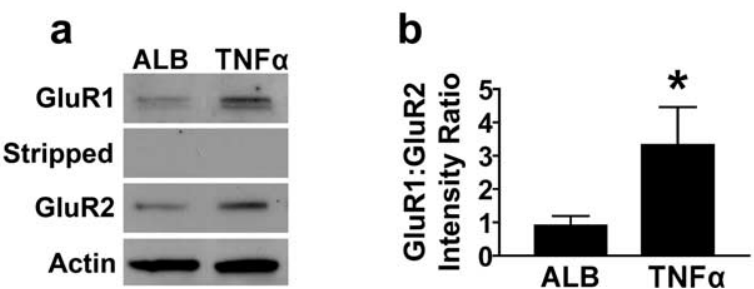

Figure 2. Biochemical evaluation of AMPAR trafficking to the plasma membrane after $\operatorname{TNF} \alpha$. A, Procedure used to inject the spinal gray matter with nanoliter quantities of TNF $\alpha$ or vehicle containing albumin. $\boldsymbol{B}$, Subcellular fractionation by low speed centrifugation yields a modest enrichment in the plasma membrane protein $\mathrm{N}$-cadherin in the P2 fraction (supplemental Fig. 1, available at www.jneurosci.org as supplemental material); however, actin is observed in all fractions. C, Densitometric analysis of Western blots from the P2 fraction reveals an increase of the GluR1:GluR2 ratio within the same blots after stripping and reprobing, suggesting a greater proportion of AMPARs that lack the GluR2 subunit in the plasma membrane after TNF $\alpha\left({ }^{*} p<0.05\right.$, Mann-Whitney U; $n=5$ subjects per group); however, actin levels did not change $(p>0.05)$. Representative examples ( $C$ reflect tissue from an albumin subject and a TNF $\alpha$ subject that were run on the same gel. Bars indicate group means of densitometry averaged across subjects. Error bars indicate SEM.

homogenized with 30 passes of a "Type B" pestle in a dounce homogenizer (Kontes) followed by 5 passes through a 22 gauge needle in ice-cold buffer, pH 7.5, containing $10 \mathrm{~mm}$ Tris, $300 \mathrm{~mm}$ sucrose, and a complete mini protease inhibitor mixture (Roche). Crude homogenate was centrifuged at $5000 \mathrm{RCF}$ for $5 \mathrm{~min}$ at $4^{\circ} \mathrm{C}$. Supernatant (S1) was further fractionated at 13,000 RCF for $30 \mathrm{~min}$. Supernatant (S2) was transferred to a new tube and pellet (P2) was resuspended in PBS (50 $\mu \mathrm{l})$ containing protease inhibitor. All samples were sonicated and stored at $-80^{\circ} \mathrm{C}$ for later processing. $\mathrm{N}$-cadherin was used to confirm plasma membrane enrichment (supplemental Fig. 1, available at www.jneurosci.org as supplemental material).

Protein assay and immunoblotting. Total protein was determined with BCA (Pierce) and quantified with a plate reader (Tecan; GeNios). Samples from each subject were separately mixed with cold Laemmli sample buffer $(62.5 \mathrm{~mm}$ Tris $\mathrm{HCl}, \mathrm{pH}$ 6.8, 25\% glycerol, 2\% SDS, 5\% $\beta$-mercaptoethanol) and immediately loaded into precast $12.5 \%$ Tris$\mathrm{HCl}$ polyacrylamide gels (Bio-Rad). Samples from albumin- and TNF $\alpha$ treated subjects were loaded pairwise on adjacent lanes $(40 \mu \mathrm{g} / \mathrm{lane})$ in a semirandomized manner to counterbalance for regional variability within the gel. To minimize potential for bias, the experimenter was unaware of condition during gel loading. Spinal cord fractions and a positive control of brain homogenate (supplemental Fig. 1, available at www.jneurosci.org as supplemental material) were electrophoresed in SDS running buffer (Bio-Rad; 25 mm Tris, $192 \mathrm{~mm}$ glycine, $0.1 \%$ SDS, pH 8.3). Molecular weights were confirmed with kaleidoscopic precision protein standard (Bio-Rad). Proteins were transferred to nitrocellulose membrane in cold tris-glycine buffer (25 mM Tris, $192 \mathrm{~mm}$ glycine, 20\% methanol, $\mathrm{pH}$ 8.3). Membranes were blocked for $1 \mathrm{~h}$ at room temperature with $5 \%(\mathrm{w} / \mathrm{v})$ nonfat dry milk in Tween-tris-buffered saline (TTBS) [0.1\% (v/v) Tween 20, $50 \mathrm{~mm}$ Tris, $150 \mathrm{~mm} \mathrm{NaCl}, \mathrm{pH}$ 7.6] and then incubated overnight at $4^{\circ} \mathrm{C}$ with primary antibodies in $1 \%$ milk TTBS. Membranes were washed $3 \times 10 \mathrm{~min}$ with TTBS and then incubated with goat anti-rabbit or goat anti-mouse horseradish peroxidase-conjugated antibodies (Pierce) for $1 \mathrm{~h}$ at room temperature in $1 \%$ milk TTBS. Membranes were washed $3 \times 10 \mathrm{~min}$ with TTBS $(0.05 \%$ Tween), incubated with ECL substrate (West Femto; Pierce), and exposed to x-ray film (Phenix). After blotting GluR1, membranes were stripped with $0.2 \mathrm{~N}$ $\mathrm{NaOH}$ for 5 min, rinsed with nanopure water and then reprobed for GluR2. A second stripping step was performed before probing with $\mathrm{N}$-cadherin. The success of stripping was confirmed by ECL. Actin (BD transduction laboratories) was probed as a loading control after $\mathrm{N}$-cadherin visualization (supplemental Fig. 1, available at www. jneurosci.org as supplemental material).

Densitometry. Films were digitized using a Perfection 1200 photo scanner (Epson) and quantified with Kodak 1d software. Bands were detected in an automated manner by the software with supervision by experimenter who was unaware of experimental condition. Mean intensity of each band was measured relative to the mean background immediately above and below the band. Exposure time was optimized for each protein to maximize the difference between band intensity and background. To control for potential loading-induced variance in total AMPAR levels while remaining sensitive to differential changes in subunit composition, the GluR1:GluR2 intensity ratio was used as the primary outcome. Statistical analysis of the actin density was used to confirm equal total protein loading.

Histological processing. For imaging experiments, subjects were killed under deep anesthesia by transcardiac perfusion with $0.9 \%$ saline followed by $4 \%$ paraformaldehyde. Lengths of spinal cord $(30 \mathrm{~mm})$ centered on the injection sites or lesion were removed and postfixed overnight $(<18 \mathrm{~h})$ in $4 \%$ paraformaldehyde. Tissue was cryoprotected in $30 \%$ sucrose for $2 \mathrm{~d}$, cut into $10 \mathrm{~mm}$ blocks, flash frozen on dry ice, and placed in a $-80^{\circ} \mathrm{C}$ freezer for later processing. Tissue was embedded in OCT and sectioned $20 \mu \mathrm{m}$ horizontally, dividing adjacent sections across four sets of slides.

Immunohistochemistry. Antibody labeling was performed on fixed tissue sections from a full set of experimental conditions simultaneously using a high-throughput staining station (Sequenza; Thermo Scientific). Before antibody application, tissue was blocked and permeabilized for $1 \mathrm{~h}$ with $5 \%$ normal goat serum and $0.3 \%$ Triton X-100. Sections were incubated overnight at room temperature with a solution containing mouse monoclonal antibody for presynaptic synaptophysin (1:200) and rabbit polyclonal anti-AMPAR antibodies $(1: 200)$ directed at the C-terminal intracellular domains of GluR1 or GluR2 (Millipore). cFos was labeled with a rabbit primary (Oncogene) at 1:5000 coincubated with synaptophysin to identify neurons. GluR1, GluR2, and cFos were applied to serial slide sets allowing regional specificity of the effects to be compared within a given subject. After a wash with $2 \mathrm{ml}$ of PBS, all slides were incubated for $1 \mathrm{~h}$ at RT with the same freshly diluted fluorescent secondary antibody solution containing 1:100 Alexa 488 goat anti-rabbit (Invitrogen) and Alexa 633 goat-anti-mouse. These procedures labeled AMPARs or cFos as Alexa 488 and synaptophysin as Alexa 633. Slides were briefly rinsed with $2 \mathrm{ml}$ PBS and coverslipped with Vectashield containing DAPI (4',6-diamidino-2-phenylindole) (Vector Laboratories). Each immunolabel had three negative controls: no primary, and each individual primary with the incorrect secondary. Confocal microscopy on these controls revealed virtually no detectable label above threshold.

Unbiased confocal sampling procedures. For contusion tissue, a region of interest was selected using c-Fos within the penumbra of the expanding lesion. Although motoneurons do not label strongly for c-Fos, labeling in smaller neurons (Fig. $1 B$ ) was used as a bioassay for activation in the region of imaged motoneurons. Confocal stacks were sampled within the c-fos positive region both rostral and caudal to the injury site. Using microscope stage coordinates, control samples were selected to be di- 
rectly contralateral to injury samples. For nanoinjection experiments, one large motoneuron was sampled every $100 \mu \mathrm{m}$ along the rostrocaudal axis of each ventral horn in horizontal sections for a $600 \mu \mathrm{m}$ length of spinal cord rostral and caudal to the injection center (supplemental Fig. 2 , available at www.jneurosci.org as supplemental material). For all experiments, large ventral motoneurons (defined by diameter $>40 \mu \mathrm{m}$ ) were selected under wide-field fluorescence by researchers who were blinded to experimental condition. Cells were selected by the characteristic pattern of presynaptic synaptophysin outlining the plasma membrane, thereby ensuring that researchers were blinded to the postsynaptic AMPA receptor levels at the time of cell selection. If several motoneurons existed at a given sampling site, a single cell was selected at random.

Laser scanning confocal microscopy and deconvolution. Confocal stacks were generated for large motoneurons using a Zeiss 510 META laser scanning confocal microscope with a $63 \times$ objective $(\mathrm{NA}=1.4)$ and a $2 \times$ zoom. Bandpass filter and laser settings were optimized on control tissue and then held constant for the duration of the experiment, providing consistent, near-complete spectral separation of synaptophysin (Alexa 633), FluoroRuby (Texas Red), and AMPARs (Alexa 488). Confocal slices $(1 \mu \mathrm{m})$ over-sampled at $0.5 \mu \mathrm{m}$ z-intervals were deblurred using 3D-blind iterative deconvolution (AutoQuant). Iteration number for the deconvolution algorithm was determined using a random subset of images and then held constant for each label for the duration of the study (iteration, 2 for GluR1; 4 for GluR2). Sequential use of confocal and deconvolution allowed higher resolution than achievable by either technique alone, providing more precise localization of receptor puncta (supplemental Fig. 2, available at www.jneurosci.org as supplemental material).

Automated confocal image analysis. MetaMorph software (Molecular Devices) was used to quantify the number of fluorescently labeled receptor puncta on the plasma membrane using custom-designed macro programs. All measures represent the number of pixels above a predetermined threshold that was established on control tissue and then held constant throughout the study. The first macro measured, for the threedimensional z-series of each field, the total number of AMPAR positive pixels (reflecting both membrane and intracellular receptors) and AM$\mathrm{PAR} /$ synaptophysin colocalized pixels (reflecting synaptic AMPARs in the neuropil). Similar measurement procedures have been shown to be sensitive to subcellular localization of receptors in vitro (Beattie et al., 2002). A second macro screened confocal stacks to identify the optical section with maximal synaptophysin/AMPAR colocalization. A blinded researcher supervised the algorithm, dropping planes that were inappropriately selected based on staining artifacts. The motoneuron plasma membrane was traced on the selected optical plane using the synaptophysin channel and a subroutine generated a $2 \mu \mathrm{m}$-wide cutout representing plasma membrane area (see Fig. 4C,D). MetaMorph then measured the proportion of this plasma membrane area labeled with AMPAR puncta alone (representing extrasynaptic receptors) and colocalized with synaptophysin (representing synaptic receptors).

Nissl staining. Nissl staining was performed using $0.5 \%$ cresyl violet (Sigma) that was dissolved in $\mathrm{dH}_{2} \mathrm{O}$ and filtered. Tissue sections from each experimental condition were mounted on the same slide, and staining was performed on the entire set at the same time, thereby minimizing systematic variance in staining. Tissue was briefly rinsed in $\mathrm{dH}_{2} \mathrm{O}$ and then stained with cresyl violet for $10 \mathrm{~min}$. After a 5 min wash in $95 \%$ $\mathrm{EtOH}$, tissue was differentiated in $95 \% \mathrm{EtOH}$ containing a small amount of glacial acetic acid (10 drops/180 ml). Differentiation was checked under a microscope by a researcher who was blinded to experimental condition. Tissue was serially washed in $100 \% \mathrm{EtOH}(2 \times 3 \mathrm{~min})$, serially placed in Xylene $(4 \times 3 \mathrm{~min})$, and coverslipped with DPX mounting medium (Sigma).

Robotic brightfield microscopy and automated cell counts. To quantify the extent of sparing after drug treatment we used a slide scanning system mounted on a Zeiss Axioplan 2 microscope. A motorized stage under precise digital feedback control allowed us to build, in an automated manner, full montage reconstructions of horizontal tissue sections through the ventral horn from high-power images (see Figs. 5, 6). We then designed an image analysis algorithm that counted all Nissl-positive objects above a preset threshold within the tissue section using a moving,
$100 \mu \mathrm{m}$ sampling window. This produced $>367,000$ data points that were exported into SPSS statistical software where the data were filtered by size to generate counts for Nissl-positive cells in the ventral horn with a mean diameter $>40 \mu \mathrm{m}$. Verification by expert histologists (MSB and JCB) indicated that the selected cells met morphological and size criteria of large ventral motoneurons.

Statistical analyses. Immunofluorescence data were analyzed using mixed ANOVA (injection side entered as a within-subject variable) and analysis of covariance (ANCOVA) to control for overall changes in the number of AMPAR-positive puncta. By statistically controlling for total AMPAR labeling with ANCOVA, we were able to distinguish receptor trafficking (subcellular redistribution) from an increase in total AMPAR puncta numbers (presumably through de novo synthesis). Densitometry data were analyzed nonparametrically with Mann-Whitney U. Brightfield data were analyzed with mixed ANOVA with distance from lesion center as a within-subject variable. For all parametric analyses, main effects and interactions were followed up with one-way ANOVA with Tukey's post hoc tests. Significance was assessed at $p<0.05$.

\section{Results}

To achieve the highest possible standards for quantitative interpretation, all experiments were performed in a fully blinded manner. Arbitrary codes were used to label drug vials, subject conditions were unknown, and subcellular fractions were coded during gel loading to eliminate possible sources of systematic bias. Conditions were only decoded after all surgery, biochemistry, microscopy, image analysis and database compilation was completed.

\section{Spinal cord injury acutely increases synaptic GluR1 within a cFos+ penumbra at the lesion edge}

Previous in vitro work has revealed that AMPAR trafficking can be detected optically by simultaneously imaging the presynaptic vesicular protein synaptophysin and AMPARs, which are expressed postsynaptically (Lissin et al., 1998; Stellwagen et al., 2005). To test whether altered AMPAR trafficking occurs in spinal cord injury we applied a similar analysis method to perfused, fixed, and permeabilized tissue sections from a preclinical model of spinal contusion injury. Accurate subcellular localization of AMPAR puncta within fixed tissue sections was achieved using a serial imaging methodology of high-resolution confocal microscopy followed by $3 \mathrm{~d}$ blind-iterative deconvolution (supplemental Fig. 2, available at www.jneurosci.org as supplemental material). Given that $\mathrm{TNF} \alpha$ levels in spinal tissue become rapidly elevated after CNS trauma (Wang et al., 1996), we hypothesized that altered AMPAR trafficking would occur at early time points after spinal cord injury. Testing this hypothesis is challenging, in part, because cells that have the most robust increases in plasma membrane AMPAR may be selectively killed, thereby obscuring detection of AMPAR trafficking. However, if changes in AMPAR trafficking occur in a temporally and spatially graded manner, then interfering with AMPAR trafficking represents a valuable therapeutic target for stopping early secondary injury and limiting expansion of the lesion. To test this important hypothesis, we used a recently established unilateral model of cervical spinal cord injury (Gensel et al., 2006). This model is unique in that it provides the uninjured contralateral side as a control for surface AMPAR levels, allowing quantitative comparisons within a single subject (Fig. $1 A$ ). At early postinjury time points, spinal contusion is marked by an area of frank lesion at the site of impact with an expanding penumbra of secondary damage that moves centrifugally over time (Crowe et al., 1997; Beattie, 2004). Based on the hypothesis that AMPAR trafficking contributes to the early wave of secondary cell death, we examined tissue just beyond the frank lesion border for evidence of AMPAR trafficking at $90 \mathrm{~min}$ 
and $3 \mathrm{~h}$ after spinal cord injury. Because TNF $\alpha$ strongly activates cFos in the region of highest glutamate vulnerability (Hermann et al., 2001), we used cFos labeling to define a region of interest for highresolution confocal imaging of AMPAR puncta. cFos labeling on the injury side was observed in a narrow ring just beyond the region of frank damage, in tissue that otherwise appeared healthy (Fig. $1 B$ ). Confocal analysis of large motoneurons in this region indicated that spinal cord injury produced a marked increase in total synaptic AMPAR within the neuropil (Fig. $1 C, D)$ as measured by a high-throughput image analysis algorithm that was sensitive to synaptic and total AMPAR levels within the tissue. Although the increase in synaptic AMPAR was significant at both postinjury time points (Fig. $1 E)(p<0.001)$, the effect was more pronounced at $90 \mathrm{~min}$ than $3 \mathrm{~h}$, mirroring the time course of early $\mathrm{TNF} \alpha$ release in the spinal cord after contusion injury (Wang et al., 1996).

Increased synaptic GluR1 after acute spinal cord injury is not attributable to an increase in total GluR1

Recent in vitro work has shown that rapid translation of AMPAR protein in the dendrites can lead to increased insertion of new GluR1 at synaptic sites in hippocampal cultures (Ju et al., 2004). To test whether spinal cord injury increases synaptic AMPARs by increasing GluR1 protein levels, we performed ANCOVA to statistically test whether variance in the total number of GluR1 puncta could account for the injury-induced increase in synaptic GluR1. ANCOVA indicated that the increase in synaptic AMPAR was not driven by a change in total cellular AMPAR $(p<$ 0.0001 after correcting for total GluR1), implicating a receptor trafficking mechanism rather than protein synthesis/degradation.

\section{TNF $\boldsymbol{\alpha}$ increases GluR2-lacking AMPARs} in the plasma membrane in vivo

To test whether TNF $\alpha$ could contribute to

SCI-induced AMPAR trafficking we delivered TNF $\alpha$ or the vehicle (albumin) to uninjured subjects and evaluated AMPAR trafficking in vivo using a biochemical assay (Fig. 2A). Modest plasma membrane enrichment via low speed centrifugation has been used to detect AMPAR trafficking in the spinal cord in vivo (Galan et al., 2004; Whitlock et al., 2006). The presence of plasma membrane was indicated by N-cadherin (Fig. $2 B$ ). Because AMPARs that contain the GluR1 subunit but lack GluR2 have been linked to increased calcium permeability (Hollmann et al., 1991) and excitotoxicity (Vandenberghe et al., 2000), we tested the relative levels of these subunits in the plasma membrane using a stripping-reprobing protocol. Sequential immunolabeling of the same Western blots (Fig. $2 C$ ) revealed a significant increase in the GluR1:GluR2 ratio in the plasma membrane $(p<0.05$, Mann-

\section{Synaptic AMPAR Levels in Ipsilateral Spinal Neuropil 60 min after TNFa Nanoinjection}
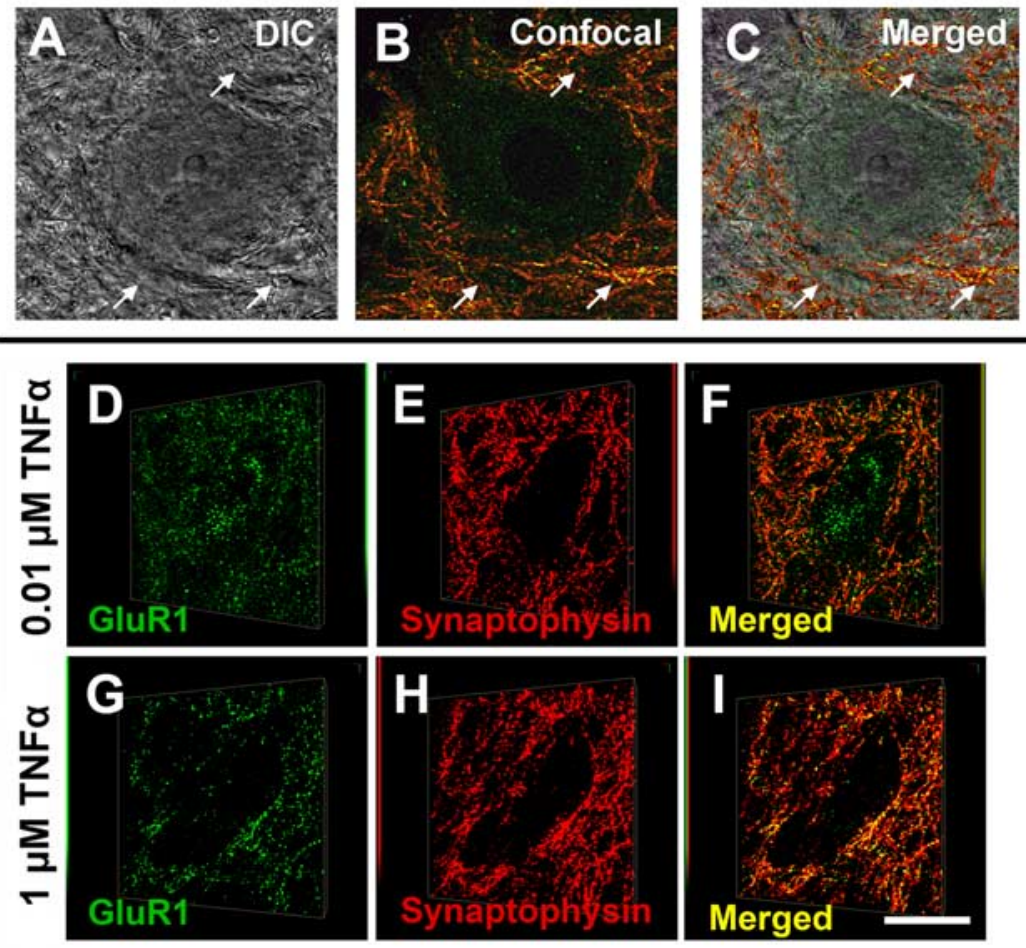

Figure 3. Increased synaptic AMPAR expression in the neuropil $60 \mathrm{~min}$ after TNF $\alpha$. A-C, Comparison of DIC and laser scanning confocal microscopy reveals synaptophysin-lined structures in the neuropil, many of which have morphology typical of large synaptic GluR1 in the neuropil after TNF $\alpha . \boldsymbol{J}, \boldsymbol{K}$, Automated quantification through the confocal stack of each cell reveals a subjects per group). Error bars indicate SEM. See supplemental Figure 3, available at www.jneurosci.org as supplemental material, for higher-order interactions. Scale bar, $30 \mu \mathrm{m}$.

Whitney $\mathrm{U}$ ), indicating an increase in the proportion of GluR2 lacking receptors in TNF $\alpha$-treated subjects.

TNF $\alpha$ increases GluR1 but not GluR2 levels at synaptic sites in the spinal neuropil in vivo

Although the biochemical fractionation assay revealed in vivo trafficking of GluR2-lacking AMPARs by TNF $\alpha$, it leaves two important questions unanswered: (1) Does TNF $\alpha$-induced AMPAR trafficking occur in clinically relevant populations of neurons? (2) Does TNF $\alpha$ induce divergent effects on GluR1 and GluR2 subunits within the same neurons, thereby predisposing these cells to excitotoxic cell death? To address these issues we performed immunohistochemistry and high resolution image analysis of large ventral horn motoneurons. These cells are exquisitely sensitive to excitotoxicity (Vandenberghe et al., 2000), 


\section{Dose-Dependent Effects at Extrasynaptic and Synaptic Plasma Membrane Sites $60 \mathrm{~min}$ After TNFa Nanoinjection in vivo}

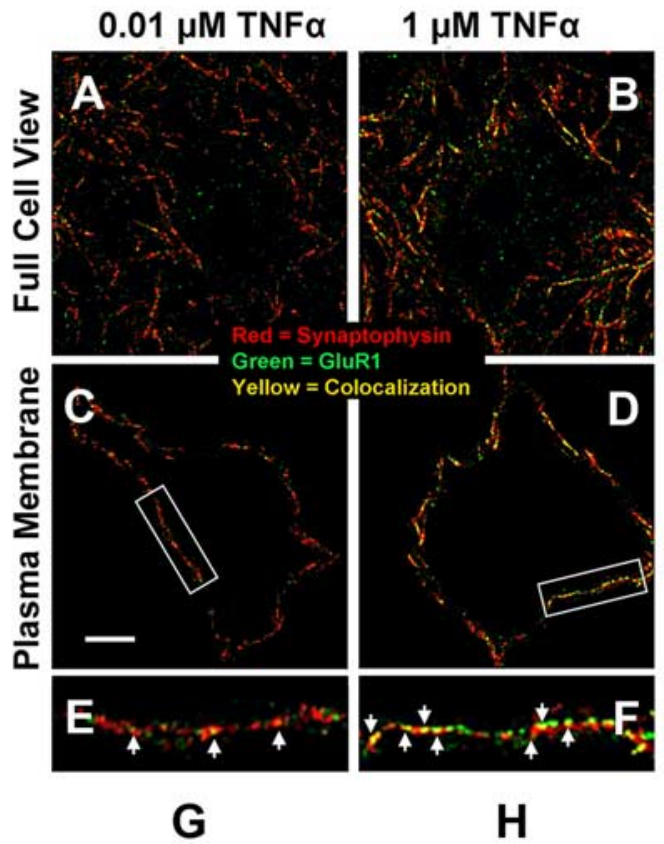

Extrasynaptic GluR1

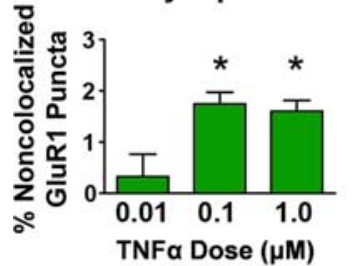

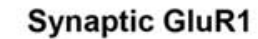

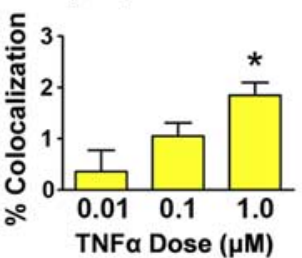

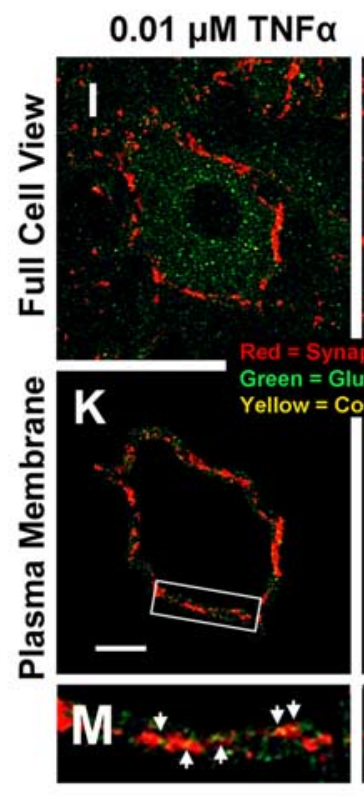

0

Extrasynaptic GluR2

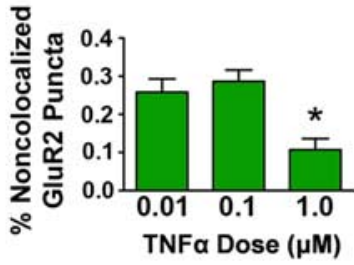

$1 \mu \mathrm{M}$ TNF $\alpha$

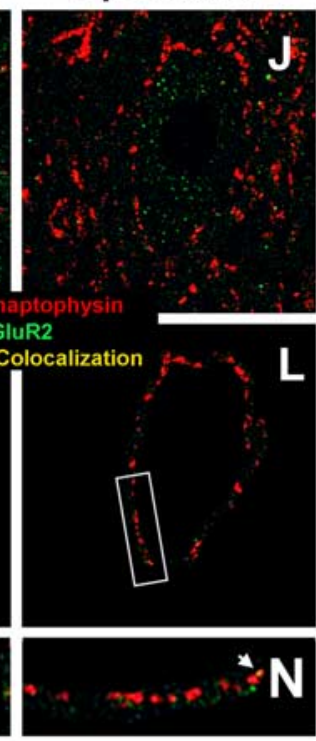

$\mathbf{P}$
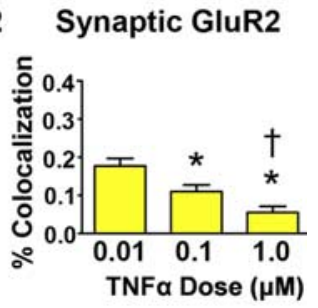

Figure 4. Increased plasma membrane localization of GluR2-lacking AMPARs on the somata of spinal motoneurons after in vivo application of TNF $\alpha$. $\boldsymbol{A}, \boldsymbol{B}$, Automated image analysis software identified, for each motoneuron, the single confocal plane with greatest colocalization of GluR1 and synaptophysin. $\boldsymbol{C}, \mathbf{D}, \mathrm{A}$ blind rater traced the cell-boundary, generating a $2 \mu \mathrm{m}$ wide optical fraction containing somatic plasma membrane. $\boldsymbol{E}, \boldsymbol{F}$, Enlargements of boxed regions illustrate mean plasma membrane levels of extrasynaptic ( $g r e e n)$ and synaptic (colocalization in yellow) GluR1 puncta after two different TNF $\alpha$ doses. $\mathbf{G}, \boldsymbol{H}$, Quantification of GluR1 levels at extrasynaptic and synaptic sites in the plasma membrane. I-N, Representative micrographs showing GluR2 levels after two different doses of TNF $\alpha$. O,P, Quantification of GluR2 at extrasynaptic and synaptic sites in the plasma membrane. TNF $\alpha$ simultaneously increased GluR1 and decreased in GluR2 in the plasma membrane in a dose-dependent manner ( ${ }^{*} p<0.05$ from lowest dose; ${ }^{\dagger} p<0.05$ from middle dose). Bars represent group means from 739 cells ( 12 animals; $n=4$ subjects per group). Error bars indicate SEM. See supplemental Figure 3, available at www.jneurosci.org as supplemental material, for higher-order interactions. Scale bars, $10 \mu \mathrm{m}$.

and their loss contributes to functional deficits in spinal cord injury and amyotrophic lateral sclerosis (Grossman et al., 2001b; Sun et al., 2006). Subunit levels were evaluated using a modification of the imaging procedures that we used to evaluate AMPAR trafficking after SCI (Fig. 1). TNF $\alpha$ and vehicle (albumin) were delivered on contralateral sides within the same subjects (Fig. 3). FluoroRuby was included in the injection solutions, providing a region of interest for high-resolution confocal microscopy (supplemental Fig. 2, available at www.jneurosci.org as supplemental material). Differential regulation of GluR1 levels at somatic and dendritic synapses has been reported in hippocampal cell culture (Grooms et al., 2006). If TNF $\alpha$ alters synaptic AMPAR levels in motoneuron dendrites, this would indicate a greater number of surface receptors available for glutamate binding, and therefore, greater potential for excitotoxicity. Superimposition of differential interference contrast (DIC) microscopy (Fig. 3A) and confocal images (Fig. $3 B$ ) revealed that many AMPAR-positive synapses existed on structures within the neuropil that have the morphology of cross-cut dendrites (Fig. 3C). To test whether $\mathrm{TNF} \alpha$-altered AMPAR levels at these synapses in vivo, we used the same image analysis algorithm that we developed to quantify colocalization of AMPARs and synaptophysin within the threedimensional confocal field after SCI. Analysis revealed that TNF $\alpha$ increased the total levels of synaptic GluR1 in a dose-dependent manner $(p<0.001)$ (Fig. 3D-J), whereas GluR2 did not change (Fig. $3 K$ ), indicating an increase in GluR2-lacking receptors. At higher doses, TNF $\alpha$ increased GluR2-lacking receptors across a broad tissue region, even extending to the contralateral side where the vehicle control was injected. This effect manifested statistically as a significant dose by side interaction by mixedfactorial ANOVA $(p<0.05)$ (supplemental Fig. 3, available at www.jneurosci.org as supplemental material).

Increased synaptic GluR1 after in vivo TNF $\alpha$ is not attributable to an increase in total GluR1

ANCOVA was used to test whether increased total GluR1 protein levels were responsible for the TNF $\alpha$-induced enhancement of synaptic GluR1. The dose-dependent effect of TNF $\alpha$ on synaptic GluR1 was robustly maintained after correcting for total protein levels ( $p<0.0001)$, suggesting that the effect of TNF $\alpha$ on synaptic GluR1 was specifically driven by redistribution of AMPARs to synaptic sites rather than an increase in total GluR1. These data 
AMPAR Trafficking in SCl after Treatment with sTNF $\alpha$ R1 A
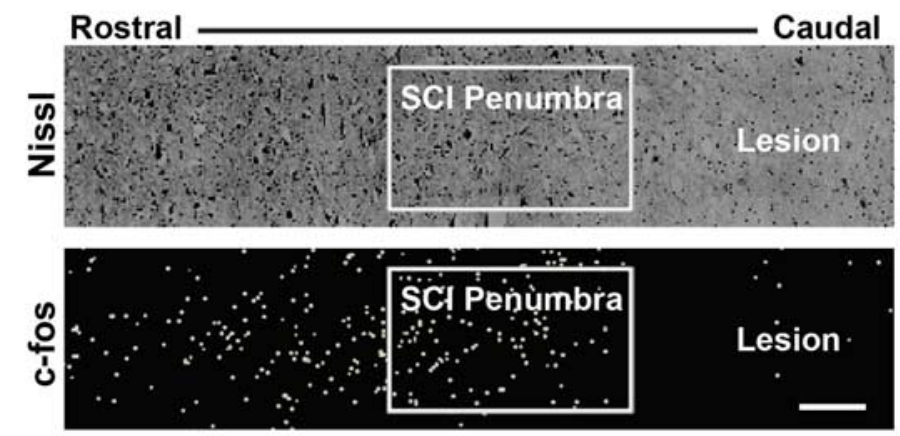
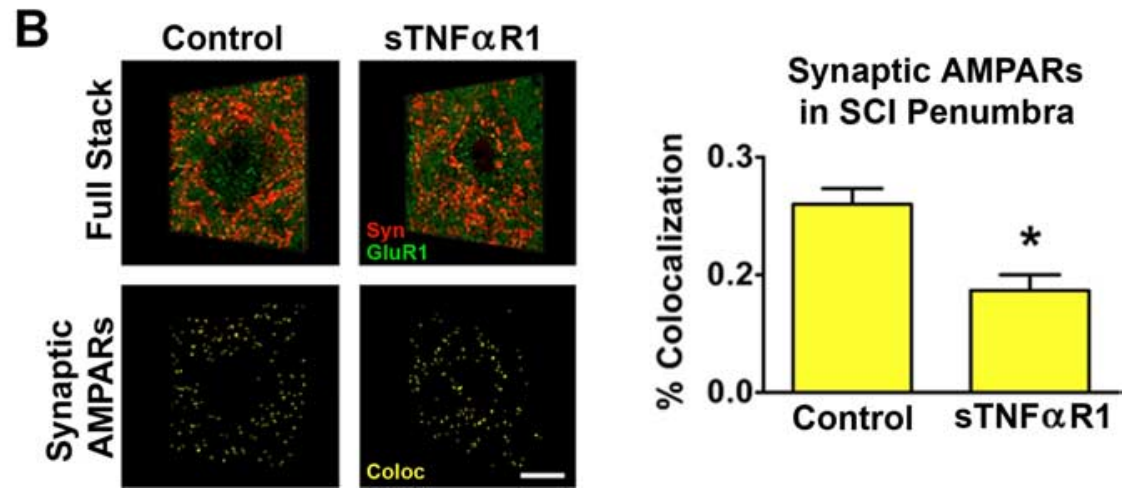

Figure 5. Treatment with sTNF $\alpha \mathrm{R} 1$ reduces injury-induced increases in synaptic AMPARs. $\boldsymbol{A}$, Representative montages of adjacent tissue sections stained for Nissl and cFos, identifying the fos-positive SCI penumbra beyond the region of frank lesion. $\boldsymbol{B}$, Representative high-resolution confocal stacks of GluR1/synaptophysin colocalization within the c-fos positive SCI penumbra after vehicle control or STNF $\alpha \mathrm{R} 1$ treatment. C, Quantitative results from algorithmic image analysis revealing reduced numbers of synaptic AMPARs in the spinal neuropil after treatment of $\mathrm{SCl}$ with sTNF $\alpha \mathrm{R} 1{ }^{*} p<0.05$ from vehicle and injury alone control conditions). Error bars indicate SEM.

indicate that the targets for therapeutic intervention may be the specific mechanisms underlying AMPAR trafficking rather than mechanisms in control of protein synthesis and degradation, just as we saw in the spinal cord injury experiments above.

\section{GluR1:GluR2 ratios change on the somatic membrane of} identified motoneurons after TNF $\alpha$ injection

To test whether TNF $\alpha$ affected the relative levels of GluR1 and GluR2 on the plasma membrane of a single identified cellular population we developed an image analysis subroutine that generated an "optical fraction" of the somatic plasma membrane of large ventral motoneurons (Fig. $4 C, D, K-L$ ). By analyzing this optical fraction, we were able to test the dose-response features of extrasynaptic and synaptic AMPAR receptors on the somatic plasma membrane of motoneurons after in vivo TNF $\alpha$.

TNF $\alpha$ induced a significant dose-dependent increase in the number of GluR1 puncta at both extrasynaptic and synaptic sites in the somatic plasma membrane $(p=0.015$ and $p<0.001$, respectively) (Fig. $4 A-H$ ). Analysis of GluR2 levels in the same motoneuron pools revealed a concurrent dose-dependent decrease in both extrasynaptic and synaptic GluR2 puncta on the somata ( $p<0.01$ and $p<0.0001$, respectively) (Fig. $4 I-P)$. Post hoc comparisons revealed that the effect on GluR1 reached statistical significance at a lower dose in extrasynaptic membrane than in synaptic membrane, suggesting that extrasynaptic insertion of GluR1-containing receptors is a prevalent feature produced by TNF $\alpha$ in the spinal cord in vivo. This is consistent with in vitro findings that AMPARs are initially inserted at extrasynaptic sites and then move into synapses through lateral trafficking (Adesnik et al., 2005). The high levels of glutamate released after SCI are likely to overwhelm peri-synaptic glutamate reuptake mechanisms, so both extrasynaptic and synaptic receptors may contribute to excitotoxicity.

Postinjury treatment with soluble TNF $\alpha$ receptor 1 reduces synaptic GluR1 within the cFos + SCI penumbra To test whether TNF $\alpha$ contributes to AMPAR trafficking after spinal cord injury, we nanoinjected a soluble form of human recombinant $\mathrm{TNF} \alpha$ receptor (sTNF $\alpha \mathrm{R} 1)$ to reduce functional $\mathrm{TNF} \alpha$ levels acutely after a unilateral cervical spinal cord injury. Synaptic AMPAR levels were measured by confocal analysis of the penumbra as identified by registering GluR1/synaptophysin-labeled sections to adjacent dorsal and ventral sections which were respectively labeled for Nissl and cFos. As observed in the first experiment (Fig. $1 B$ ), cFos labeling on the injury side occurred in a ring just beyond the region of frank damage, in tissue that otherwise appeared healthy (Fig. 5A). Blinded digital image analysis revealed that the diameter of the cFos-positive ring was not significantly altered by $s \mathrm{TNF} \alpha \mathrm{R} 1$ treatment $(p>0.05)$, confirming that cFos positivity could serve as a consistent landmark for identifying the lesion penumbra. Highresolution confocal analysis of GluR1 and synaptophysin suggested that $\mathrm{sTNF} \alpha \mathrm{R} 1$ nanoinjection reduced synaptic AMPAR levels in the neuropil after SCI (Fig. 5B). Automated quantification through the three-dimensional stacks of large motoneurons revealed a significant reduction of synaptic AMPAR numbers in sTNF $\alpha \mathrm{R} 1$ treatment relative to control conditions (albumin vehicle and noninjected SCI controls). This effect was significant after correcting for the total GluR1 numbers with ANCOVA, suggesting that sTNF $\alpha$ R1 altered AMPAR trafficking to synapses without directly impacting total GluR1 protein levels $(p<0.001)$. These data strongly suggest that TNF $\alpha$ contributes to SCI-induced trafficking of AMPARs to the synaptic plasma membrane, providing a potential therapeutic target for reducing excitotoxic cell death after CNS trauma.

Postinjury treatment with soluble TNF $\alpha$ receptor 1 reduces acute secondary excitotoxicity in SCI

To test the therapeutic potential of interfering with TNF $\alpha$ induced AMPAR trafficking after CNS trauma, we tested the effect of sTNF $\alpha$ R 1 on motoneuron sparing after SCI. An automated cell counting algorithm revealed significant sparing of Nissl-positive large motoneurons after $\mathrm{sTNF} \alpha \mathrm{R} 1$ treatment within a region of penumbra that was selected in a blinded, a priori manner (Fig. 6A). Representative examples of microscope fields within the penumbra sample window illustrate the increase in spared numbers of large (diameter $>40 \mu \mathrm{m}$ ) ventral neurons that met the thresholding and size criteria of the automated algorithm (Fig. 6B). One-way ANOVA and post hoc tests revealed a significant increase in spared neurons within the a priori sample 
window (Fig. 6C) $(p<0.05)$ after sTNF $\alpha$ R1 treatment. The protective effect was more pronounced rostral to the injury site and was evident by 90 min after injury, the same time point at which SCI increased synaptic AMPAR levels were found to be elevated in the absence of sTNF $\alpha$ R1 (Fig. 4).

\section{Discussion}

After SCI, TNF $\alpha$ levels become elevated in the spinal cord, reaching peak levels within $1 \mathrm{~h}$ after initial trauma (Wang et al., 1996). Within the same time period, large numbers of neurons start to die through an excitotoxic mechanism. The present findings suggest that TNF $\alpha$-induced trafficking of AMPA receptors contributes to this excitoxic cell death after spinal cord injury in vivo. We evaluated AMPAR trafficking in two in vivo models of spinal cord injury. We first tested for SCI-induced AMPAR trafficking using a hemi-contusion model of cervical spinal cord injury that produces a focal lesion with associated unilateral functional deficits (Gensel et al., 2006). The data revealed an increase in AMPAR numbers at synapses ipsilateral to the lesion at $90 \mathrm{~min}$ and $3 \mathrm{~h}$ after injury that was not accounted for by an increase in total GluR1, suggesting an AMPAR-trafficking mechanism. To test whether injuryinduced $\mathrm{TNF} \alpha$ may contribute to this AMPAR trafficking in the spinal cord in vivo, we nanoinjected TNF $\alpha$ into the ventral horns and performed biochemical and optical analysis of the tissue at 60 min after injection (supplemental Figs. 1, 2, available at www.jneurosci.org as supplemental material). These experiments revealed that in vivo TNF $\alpha$ simultaneously increases GluR1 and decreases GluR2 at both extrasynaptic and synaptic plasma membrane sites. Given that GluR2lacking AMPARs have increased permeability to calcium (Cull-Candy et al., 2006), TNF $\alpha$-induced AMPAR trafficking is likely to contribute to postinjury excitotoxicity. Recent in vitro work has suggested that TNF $\alpha$ causes a transient exocytosis of GluR2-lacking AMPARs at extrasynaptic sites within 15 min of TNF $\alpha$ exposure in hippocampal primary culture, but then resolves by $1 \mathrm{~h}$ (Leonoudakis et al., 2008). This would suggest an unrealistic temporal window for intervention in most clinical settings, however the present findings suggest that in vivo these effects occur on a lagged timescale, offering a potential treatment opportunity. To test this therapeutic potential, we sequestered TNF $\alpha$ after cervical spinal cord injury with a soluble TNF $\alpha$ receptor and found a reduction in synaptic AMPAR, and associated reductions in excitotoxic cell death as measured by greater numbers of spared neurons after acute SCI.

To our knowledge, this is the first demonstration of $\mathrm{TNF} \alpha$ induced AMPAR trafficking in the context of an in vivo preclinical model of a CNS disorder. Glutamate receptors have long been implicated in secondary excitotoxicity in disease and after neurotrauma (Gómez-Pinilla et al., 1989; Wrathall et al., 1996); however, reports of changes in the expression of glutamate receptors have generally been concerned with long term changes in total protein levels. Here, we emphasize the more rapid posttranslational changes in excitotoxicity related to receptor traf- ficking events. Many drugs that directly antagonize glutamate receptors have profound negative side effects and have failed clinical trials (Walters et al., 2005; Chen and Lipton, 2006). The present findings suggest a novel approach for modulating glutamate excitotoxicity after spinal cord injury using drugs that act on TNF $\alpha$ signaling. This may have immediate translational benefit, as TNF $\alpha$ inhibitors have already passed clinical trials for other inflammatory syndromes such as rheumatoid arthritis. Moreover, because SCI-induced AMPAR trafficking persisted for at least $3 \mathrm{~h}$, there appears to be a relatively broad time window for therapeutic intervention.

It is important to recognize that the present findings are based on several elegant in vitro studies of hippocampal synaptic plasticity, which have revealed that TNF $\alpha$ can alter synaptic strength by modulating AMPAR trafficking (Beattie et al., 2002; Stellwagen et al., 2005; Stellwagen and Malenka, 2006; Leonoudakis et al., 2008). However, before the present findings, TNF $\alpha$-induced AMPAR trafficking had never been directly demonstrated in vivo and its role in cell death after CNS injury had not been known.

Previous work has revealed that AMPAR subunit mRNA levels do not change within the first $24 \mathrm{~h}$ after spinal cord injury (Grossman et al., 2001a), although the majority of acute motoneuron excitotoxicity occurs (Grossman et al., 2001b), and anti-TNF $\alpha$ drugs are protective (Genovese et al., 2006) within this time frame. The present findings provide an explanation for this apparent paradox, suggesting that early injury-induced excitotoxicity (Hermann et al., 2001) is attributable to rapid trafficking of AMPARs to the synaptic plasma membrane rather than changes in total cellular AMPAR numbers. At later time points $(>24 \mathrm{~h})$, changes in total AMPAR protein levels (increased GluR1:GluR2 ratio) have been reported (Grossman et al., 1999), 
and these long term changes may contribute to ongoing cell death rather than early secondary injury processes.

It is noteworthy that TNF $\alpha$ selectively increased plasma membrane levels of the GluR2-lacking receptor, an AMPAR subpopulation with increased current passing capability and greater permeability to calcium (Van den Bosch et al., 2000; Vandenberghe et al., 2000). AMPAR trafficking occurred at TNF $\alpha$ doses that potentiate excitotoxicity in the spinal cord in vivo (Hermann et al., 2001), providing a mechanism for these previous findings. Trafficking of GluR2-lacking receptors may also provide a mechanistic explanation for findings within the pain literature that TNF $\alpha$ increases excitability in cultured spinal cord sensory neurons (Schäfers et al., 2003a) and produces tactile hypersensitivity in vivo (Schäfers et al., 2003b). Thus, the implications of the present work extend beyond spinal cord pathology. Excitotoxicity through GluR2-lacking AMPARs may be a common mechanism of cell loss in a variety of neurodegenerative conditions including not only spinal cord injury but amyotrophic lateral sclerosis, multiple sclerosis, ischemic injury, and Alzheimer's disease (Agrawal and Fehlings, 1997; Pitt et al., 2000; Blanchard et al., 2004; Leonoudakis et al., 2004; Lai et al., 2006; Liu et al., 2006; Tobinick and Gross, 2008), disease states that also have an inflammatory component. By providing the first in vivo demonstration that TNF $\alpha$ regulates trafficking of GluR2-lacking AMPARs, the present findings suggest novel therapeutic targets for treating excitotoxicity in CNS injury and disease.

\section{References}

Adesnik H, Nicoll RA, England PM (2005) Photoinactivation of native AMPA receptors reveals their real-time trafficking. Neuron 48:977-985.

Agrawal SK, Fehlings MG (1997) Role of NMDA and non-NMDA ionotropic glutamate receptors in traumatic spinal cord axonal injury. J Neurosci 17:1055-1063.

Beattie EC, Stellwagen D, Morishita W, Bresnahan JC, Ha BK, Von Zastrow M, Beattie MS, Malenka RC (2002) Control of synaptic strength by glial TNF alpha. Science 295:2282-2285.

Beattie MS (2004) Inflammation and apoptosis: linked therapeutic targets in spinal cord injury. Trends Mol Med 10:580-583.

Blanchard BJ, Chen A, Rozeboom LM, Stafford KA, Weigele P, Ingram VM (2004) Efficient reversal of Alzheimer's disease fibril formation and elimination of neurotoxicity by a small molecule. Proc Natl Acad Sci U S A 101:14326-14332.

Chen HS, Lipton SA (2006) The chemical biology of clinically tolerated NMDA receptor antagonists. J Neurochem 97:1611-1626.

Crowe MJ, Bresnahan JC, Shuman SL, Masters JN, Beattie MS (1997) Apoptosis and delayed degeneration after spinal cord injury in rats and monkeys. Nat Med 3:73-76.

Cull-Candy S, Kelly L, Farrant M (2006) Regulation of Ca2+-permeable AMPA receptors: synaptic plasticity and beyond. Curr Opin Neurobiol 16:288-297.

Ehlers MD (2000) Reinsertion or degradation of AMPA receptors determined by activity-dependent endocytic sorting. Neuron 28:511-525.

Galan A, Laird JM, Cervero F (2004) In vivo recruitment by painful stimuli of AMPA receptor subunits to the plasma membrane of spinal cord neurons. Pain 112:315-323.

Genovese T, Mazzon E, Crisafulli C, Di Paola R, Muia C, Bramanti P, Cuzzocrea S (2006) Immunomodulatory effects of etanercept in an experimental model of spinal cord injury. J Pharmacol Exp Ther 316:1006-1016.

Gensel JC, Tovar CA, Hamers FP, Deibert RJ, Beattie MS, Bresnahan JC (2006) Behavioral and histological characterization of unilateral cervical spinal cord contusion injury in rats. J Neurotrauma 23:36-54.

Gómez-Pinilla F, Tram H, Cotman CW, Nieto-Sampedro M (1989) Neuroprotective effect of Mk-801 and U-50488H after contusive spinal-cord injury. Experimental Neurology 104:118-124.

Grooms SY, Noh KM, Regis R, Bassell GJ, Bryan MK, Carroll RC, Zukin
RS (2006) Activity bidirectionally regulates AMPA receptor mRNA abundance in dendrites of hippocampal neurons. J Neurosci 26:8339-8351.

Grossman SD, Wolfe BB, Yasuda RP, Wrathall JR (1999) Alterations in AMPA receptor subunit expression after experimental spinal cord contusion injury. J Neurosci 19:5711-5720.

Grossman SD, Rosenberg LJ, Wrathall JR (2001a) Relationship of altered glutamate receptor subunit mRNA expression to acute cell loss after spinal cord contusion. Exp Neurol 168:283-289.

Grossman SD, Rosenberg LJ, Wrathall JR (2001b) Temporal-spatial pattern of acute neuronal and glial loss after spinal cord contusion. Experimental Neurology 168:273-282.

Hering H, Lin CC, Sheng M (2003) Lipid rafts in the maintenance of synapses, dendritic spines, and surface AMPA receptor stability. J Neurosci 23:3262-3271.

Hermann GE, Rogers RC, Bresnahan JC, Beattie MS (2001) Tumor necrosis factor-alpha induces cFOS and strongly potentiates glutamate-mediated cell death in the rat spinal cord. Neurobiology of Disease 8:590-599.

Hollmann M, Hartley M, Heinemann S (1991) Ca2+ permeability of Kaampa gated glutamate receptor channels depends on subunit composition. Science 252:851-853.

Ju W, Morishita W, Tsui J, Gaietta G, Deerinck TJ, Adams SR, Garner CC, Tsien RY, Ellisman MH, Malenka RC (2004) Activity-dependent regulation of dendritic synthesis and trafficking of AMPA receptors. Nat Neurosci 7:244-253.

Lai C, Xie C, McCormack SG, Chiang HC, Michalak MK, Lin X, Chandran J, Shim H, Shimoji M, Cookson MR, Huganir RL, Rothstein JD, Price DL, Wong PC, Martin LJ, Zhu JJ, Cai H (2006) Amyotrophic lateral sclerosis 2-deficiency leads to neuronal degeneration in amyotrophic lateral sclerosis through altered AMPA receptor trafficking. J Neurosci 26:11798-11806.

Leonoudakis D, Braithwaite SP, Beattie MS, Beattie EC (2004) TNF-alphainduced AMPA-receptor trafficking in CNS neurons; relevance to excitotoxicity? Neuron Glia Biol 1:263-273.

Leonoudakis D, Zhao P, Beattie EC (2008) Rapid tumor necrosis factor alpha-induced exocytosis of glutamate receptor 2-lacking AMPA receptors to extrasynaptic plasma membrane potentiates excitotoxicity. J Neurosci 28:2119-2130.

Lissin DV, Gomperts SN, Carroll RC, Christine CW, Kalman D, Kitamura M, Hardy S, Nicoll RA, Malenka RC, von Zastrow M (1998) Activity differentially regulates the surface expression of synaptic AMPA and NMDA glutamate receptors. Proc Natl Acad Sci U S A 95:7097-7102.

Liu B, Liao M, Mielke JG, Ning K, Chen Y, Li L, El-Hayek YH, Gomez E, Zukin RS, Fehlings MG, Wan Q (2006) Ischemic insults direct glutamate receptor subunit 2-lacking AMPA receptors to synaptic sites. J Neurosci 26:5309-5319.

Malinow R, Malenka RC (2002) AMPA receptor trafficking and synaptic plasticity. Annu Rev Neurosci 25:103-126.

Pitt D, Werner P, Raine CS (2000) Glutamate excitotoxicity in a model of multiple sclerosis. Nat Med 6:67-70.

Schäfers M, Lee DH, Brors D, Yaksh TL, Sorkin LS (2003a) Increased sensitivity of injured and adjacent uninjured rat primary sensory neurons to exogenous tumor necrosis factor-alpha after spinal nerve ligation. J Neurosci 23:3028-3038.

Schäfers M, Svensson CI, Sommer C, Sorkin LS (2003b) Tumor necrosis factor-alpha induces mechanical allodynia after spinal nerve ligation by activation of p38 MAPK in primary sensory neurons. J Neurosci 23:2517-2521.

Sekine-Aizawa Y, Huganir RL (2004) Imaging of receptor trafficking by using alpha-bungarotoxin-binding-site-tagged receptors. Proc Natl Acad Sci U S A 101:17114-17119.

Skeberdis VA, Lan J, Zheng X, Zukin RS, Bennett MV (2001) Insulin promotes rapid delivery of $\mathrm{N}$-methyl-D-aspartate receptors to the cell surface by exocytosis. Proc Natl Acad Sci U S A 98:3561-3566.

Stellwagen D, Malenka RC (2006) Synaptic scaling mediated by glial TNFalpha. Nature 440:1054-1059.

Stellwagen D, Beattie EC, Seo JY, Malenka RC (2005) Differential regulation of AMPA receptor and GABA receptor trafficking by tumor necrosis factor-alpha. J Neurosci 25:3219-3228.

Sun H, Kawahara Y, Ito K, Kanazawa I, Kwak S (2006) Slow and selective death of spinal motor neurons in vivo by intrathecal infusion of kainic 
acid: implications for AMPA receptor-mediated excitotoxicity in ALS. J Neurochem 98:782-791.

Tobinick EL, Gross H (2008) Rapid cognitive improvement in Alzheimer's disease following perispinal etanercept administration. J Neuroinflammation 5:2.

Vandenberghe W, Robberecht W, Brorson JR (2000) AMPA receptor calcium permeability, GluR2 expression, and selective motoneuron vulnerability. J Neurosci 20:123-132.

Van Den Bosch L, Vandenberghe W, Klaassen H, Van Houtte E, Robberecht W (2000) Ca2+-permeable AMPA receptors and selective vulnerability of motor neurons. J Neurol Sci 180:29-34.

Walters MR, Kaste M, Lees KR, Diener HC, Hommel M, De Keyser J, Steiner
H, Versavel M (2005) The AMPA antagonist ZK 200775 in patients with acute ischaemic stroke: a double-blind, multicentre, placebo-controlled safety and tolerability study. Cerebrovasc Dis 20:304-309.

Wang CX, Nuttin B, Heremans H, Dom R, Gybels J (1996) Production of tumor necrosis factor in spinal cord following traumatic injury in rats. J Neuroimmunol 69:151-156.

Whitlock JR, Heynen AJ, Shuler MG, Bear MF (2006) Learning induces long-term potentiation in the hippocampus. Science 313:1093-1097.

Wrathall JR, Teng YD, Choiniere D (1996) Amelioration of functional deficits from spinal cord trauma with systemically administered NBQX, an antagonist of non-N-methyl-D-aspartate receptors. Exp Neurol 137:119-126. 